\title{
Balkanologie
}

Balkanologie Revue d'études pluridisciplinaires

Vol. IX, n' 1-2 | 2005

Volume IX Numéro 1-2

\section{La magie chez les musulmans des Balkans (III) : l'apport de Tihomir R. Djordjević (1868-1944)}

Magic among Muslims in the Balkans (III): the contribution of Tihomir R.

Djordjević (1868-1944)

\section{Alexandre Popovic}

\section{OpenEdition}

\section{Journals}

Édition électronique

URL : http://journals.openedition.org/balkanologie/601

DOI : 10.4000/balkanologie.601

ISSN : 1965-0582

Éditeur

Association française d'études sur les Balkans (Afebalk)

Édition imprimée

Date de publication : 1 décembre 2005

ISSN : 1279-7952

\section{Référence électronique}

Alexandre Popovic, "La magie chez les musulmans des Balkans (III) : l'apport de Tihomir R. Djordjević (1868-1944) », Balkanologie [En ligne], Vol. IX, n 1-2 | 2005, mis en ligne le 14 janvier 2010, consulté le 17 décembre 2020. URL : http://journals.openedition.org/balkanologie/601 ; DOI : https://doi.org/ 10.4000/balkanologie.601

(c) Tous droits réservés 
Balkanologie IX (1-2), décembre 2005 289

\section{RECHERCHES}




\title{
LA MAGIE CHEZ LES MUSULMANS DES BALKANS [III] : L'APPORT DE TIHOMIR R. DJORDJEVIĆ (1868-1944)
}

\author{
Alexandre Popovic*
}

Travaillant depuis novembre 2000 , dans le cadre de mon séminaire de l'École des Hautes Études en Sciences Sociales, sur la magie chez les musulmans balkaniques ${ }^{1}$, je me suis rapidement rendu compte de l'intérêt qu'il y avait à mener cette étude selon trois principes : (a) l'entreprendre pays par pays afin d'analyser plus facilement la quasi totalité des publications connues et de la documentation accessible ; ensuite (b) le faire en comparaison constante avec la magie pratiquée chez les populations non musulmanes du pays en question; mais aussi (c) avec la magie pratiquée dans d'autres régions du monde musulman et, avant tout - pour des raisons évidentes - avec celles des populations de l'Empire ottoman et de la Turquie moderne. Il y a lieu d'ajouter que j'ai débuté mes recherches par les territoires de l'ex-Yougoslavie, car les publications et la documentation en général y sont infiniment plus nombreuses et plus riches par rapport à celles concernant les autres pays des Balkans.

Les travaux publiés touchant à la magie chez les musulmans des territoires ex-yougoslaves sont l'œuvre d'une cinquantaine d'auteurs et sont, évidemment, d'importance très inégale. Au sommet de cette pyramide se trouvent indiscutablement les quatre principaux spécialistes, à savoir : le fondateur de l'ethnologie scientifique serbe, Tihomir R. Djordjević (1868-1944), auquel est consacrée la présente étude ; l'historien et turcologue Gligorije/Gliša Elezović (1879-1960)² ;

\footnotetext{
* (CNRS-EHESS, Paris)

${ }^{1}$ Il s'agit du séminaire intitulé " Histoire moderne et contemporaine des musulmans balkaniques ", du Centre d'histoire du domaine turc de l'EHESS.

2 Dont nous sommes en train d'analyser les travaux sur la magie, à notre séminaire de l'EHESS, depuis le début de l'annèe scolaire 2005-2006.
} 
le médecin Stanko Sielski (1891-1958)3 ; ainsi qu'un personnage atypique et extrêmement intéressant, Muhamed Garčević (né vers 1893, et mort après 1951) 4 . Après ces quatre auteurs, on doit signaler un groupe d'une dizaine d'autres noms, tels ceux de Leopold Glück (1854-1907), Kosta Hörmann (1850-1921), Lujo Thaller (1891-1949), Vladimir Bazala (1901-1987), Josip Matasović (1892-1962), Veselin Čajkanović (1881-1946), Milovan Gavazzi (1895-1992), et le spécialiste d'une forme particulière de magie appelée bajanje (lire bayanye), Ljubinko Radenković (né en 1951)5. Finalement, il existe un dernier groupe d'auteurs moins connus, qui ont cependant eux aussi apporté leur obole à l'étude de la magie en ex-Yougoslavie, suivant leurs possibilités personnelles et les circonstances historiques du moment ${ }^{6}$.

Dans ce troisième texte de cette série d'études (les deux précédents ayant été consacrés à $M$. Garčević et St. Sielski), je voudrais présenter rapidement l'apport tout à fait capital pour l'étude de la magie chez les populations des territoires ex-yougoslaves (voire balkaniques en général), du principal spécialiste de ces questions dans la première moitié du XXème siècle, à savoir Tihomir Djordjević. En effet, celui-ci avait publié en 1938, le tout premier (et pratiquement unique) ouvrage d'envergure sur ce sujet particulier.

\section{LE PERSONNAGE ET SON GEUVRE}

Originaire de la Serbie orientale, T. R. Djordjević est né dans la petite ville de Knjaževac le 19 février 1868 . Son père, Radoslav, étant pope dans le village de Tešica, Tihomir y fait ses études primaires, qu'il va poursuivre aux lycées d'Aleksinac et de Niš, avant de s'inscrire dans le département d'histoire et de philologie de la "Grande École" (Velika Škola), c'est-à-dire de la future Université de Belgrade, études qu'il terminera cum laude en 1891. Nommé professeur, puis directeur de l'École d'instituteurs et du lycée d'Aleksinac, il y enseigne de 1891 à 1906. Entre temps, il étudie pendant un semestre à l'Université

\footnotetext{
3 J'ai terminé récemment une étude sur Stanko Sielski et ses publications sur la magie, qui devrait paraître à Belgrade en 2006, dans un volume dédié à la mémoire du turcologue Slavoljub Djindjić (1935-2000).

4 Sur M. Garčević et ses publications, voir mon article, Popovic (Alexandre), " A propos de la magie chez les musulmans des Balkans n in Bouillier (Véronique) et Servan-Schreiber (Catherine), De l'Arabie à l'Himalaya. Chemins croisés en hommage à Marc Gaborieau, Paris : Maisonneuve \& Larose, 2004.

5 Voir notamment ses deux ouvrages Radenković (Ljubinko), Narodna bajanja kod Južnih Slovena (The popular bajanja among the South Slavs), Beograd : Prosveta-Balkanološki Institut SANU, 1996 ; et Radenković (Ljubinko), Simbolika sveta u narodnoj magiji Južnih Slovena (The Symbolism of the World in the popular magic among the South Slavs), Beograd / Niš : SANU / Prosveta, 1996.

6 Je compte analyser, dans les années à venir, l'apport de chacun de ces auteurs concernant la magie chez les musulmans balkaniques, dans plusieurs articles et notices de cette série de publications.
} 
de Vienne et soutient, en 1902, à l'Université de Munich sa thèse de doctorat Die Zigeuner in Serbien (Les Tsiganes de Serbie), publiée aussitôt à Budapest (tome I dès 1903, tome II en 1906). En 1906 également, il est nommé professeur au Premier lycée de Belgrade et est élu maître assistant (docent) à l'Université.

Mobilisé pendant les Guerres balkaniques (1912-1913), puis pendant la Première Guerre mondiale, il participe à la retraite de l'armée serbe à travers l'Albanie en 1915. Envoyé en mission officielle (de 1916 à 1918) à Londres, et ensuite à Paris, pour faire des conférences publiques sur la Serbie, son histoire, sa population, son folklore, etc., il saisit cette occasion pour suivre de près l'évolution des travaux scientifiques des spécialistes européens dans le domaine de l'ethnologie et d'autres sciences humaines (Tylor, Morgan, Frazer, Doutté, Seligmann, Budge, Westermarck, Elworthy, Wellhausen, Crooke, Carra de Vaux, et quelques autres), dont il utilisera abondamment par la suite les méthodes et les résultats, pour ses propres recherches. En 1919, il est nommé professeur extraordinaire à l'Université de Belgrade puis, en 1921 (l'année où il rentre de son long séjour à l'étranger), professeur régulier, ainsi que membre correspondant de l'Académie serbe des Sciences, dont il devient membre régulier en 1938, donc l'année même de son départ à la retraite. En 1941, au moment de l'occupation allemande, il est interné dans le tristement célèbre camp de détention de Banjica, près de Belgrade, d'où il sera finalement libéré du fait de son âge avancé et de l'état de sa santé. Il meurt d'épuisement, pour ne pas dire pratiquement de faim, dans sa soixante-dix septième année, le 28 mai 1944.

Ethnologue et folklorisant avant tout, mais également historien, balkanologue, anthropologue, pédagogue, muséologue, spécialiste d'histoire culturelle, éditeur scientifique de nombreux ouvrages collectifs et de revues 7 , il a très profondement marqué de son empreinte ces diverses disciplines en Yougoslavie. La liste de ses publications contient plus de 700 titres d'ouvrages, d'articles et de compte-rendus ${ }^{8}$, dont certains avaient été écrits directement en allemand, en anglais, ou en français. La grande majorité de ces publications porte naturellement sur son principal pôle d'intérêt, c'est-à-dire sur les différents aspects de la culture traditionnelle des diverses populations de la partie orientale de l'ex-Yougoslavie, sujet sur lequel il a patiemment rassemblé et systématisé une énorme quantité de matériaux, tirés des sources les plus diverses. Il a mis au point également sa propre technique d'enquêtes sur le terrain et de collecte des données, grâce à ses fameux "questionnaires" (upitnici, sing. upit-

\footnotetext{
$7 \mathrm{Il}$ a ainsi fondé et édité, dans la petite ville d'Aleksinac, à l'âge de 31 ans (et à ses propres frais) le périodique Karadžić (1899-1903). Voir à ce sujet : Janković (Ljubica S.), " Tihomir R. Djordjević i značaj njegovog Karadžića " (T. R. Djordjević et l'importance de son "Karadžič"), Narodno Stvaralaštvo, 28, 1968.

8 On trouvera la liste la plus complète de ses publications dans : Janković (Ljubica S.), Janković (Danica S.), " Bibliografija radova Tihomira R. Djordjevića " (Bibliographie des travaux de T. R. Djordjević), in Spomenica posvećena stogodišnjici rodjenja Tihomira Djordjevića (Mélanges dédiés à T. R. Djordjević, à l'occasion du centenaire de sa naissance), Beograd : SANU, 1971.
} 
nik) et ses "directives" (uputstva, sing. uputstvo). Pour lui, un ethnologue devait être en connexion constante avec d'autres disciplines des sciences humaines, et notamment avec l'histoire, l'archéologie et l'anthropologie. C'est pourquoi on trouvera dans ces écrits, et avant tout dans son monumental ouvrage en dix volumes, intitulé " Notre vie populaire " (Naś narodni život) ${ }^{9}$, des textes extrêmement variés : sur les coutumes et les croyances populaires des Slaves du Sud (mais aussi des Turcs, des Albanais, des Valaques, des Tsiganes et même, à quelques occasions plus rares bien entendu, des Čerkesses, des Juifs et des Noirs de ces régions), sur les métiers et les corporations, les vêtements, la médecine populaire, les coutumes médicales, les méthodes et usages ayant cours dans le domaine de la technologie, la chasse, le droit coutumier, etc. Naturellement, sa façon d'analyser les phénomènes étudiés était liée à la vision évolutionniste courante pour l'époque, qui privilégie le "regard historicisant" des manifestations observées ${ }^{10}$.

\section{SES TEXTES SUR LA MAGIE ET SA MÉTHODE}

D'après ce que j'ai pu voir jusqu'à présent de ses publications, trois parmi ses textes concernent directement notre sujet : tout d'abord un bref article de quelques pages à peine, sur les talismans, intitulé Amajlije/Hamajlije, paru en 1932 et réédité en $1984^{11}$; ensuite, une étude beaucoup plus longue sur le

9 Djordjević (T. R.), Naš narodni život (Notre vie populaire), Beograd : Srpska Književna Zadruga, 1930-1934. 10 vols. ; (seconde éd., sous la réd. d'Ivan Colović), Beograd : Prosveta, 1984, 4 vols. Sur les péripéties de la première édition, voir les détails dans le tome 4 de l'èd. de 1984, pp. 289-290; et sur les différences, plus ou moins minimes, entre ces deux éditions, les explications de l'éditeur, aux pages 333-334 du mẻme tome.

10 Sur la biographie et l'œuvre scientifique de T. R. Djordjević, voir : Cajkanović (Veselin), "Djordjević Tihomir DI ", Narodna Enciklopedija srpsko-hrvatsko-slovenačka, Zagreb, 1926, t. I, p. 695 ; Fishta (Filip). "Te Akademiku Z. Tihornir R. Gjorgjeviç ", Leka, 3 (11), novembre 1931 ; Bajraktarović (Mirko R.), " $D^{I}$ Tihomir Djordjevic i njegov rad " ( $\mathrm{D}^{\mathrm{I}} \mathrm{T}$. Dj. et son travail scientifique), Glasnik Etnografskog Muzeja u Beogradu, 16, 1953 ; Janković (Ljubica), Janković (Danica S.), "Gradja o żivotu i radu Tihomjra $\mathbf{R}$. Djordjevića " (Matériaux concernant la vie et l'œuvre de T. R. Dj.), Bilten Instituta za proučavanje folklora, 3, 1955 ; Bajraktarovic (Mirko), "Djordjevic Tihomir ", Enciklopedija Jugoslavije, Zagreb, 1958, t. 3 ; Antonijević (Dragoslav)، "Naućna metoda i tehnika Tihomira Djordjevića " (La technique et la méthode scientifique de T. Dj.). Narodno Stvaralaštvo, 28, 1968 ; Nedeljković (Dušan), “ Značaj naučnog dela Tihomira Djordjevica " (l'importance de l'œuvre scientifique de T. Dj.), Narodno Stvaralaštvo, 28, 1968; Antonijevic (Dragoslav), "Tihomir Djordjević i njegovo naučno delo" (T. Dj. et son œuvre scientifique), Zbornik za drusitvene nauke Matice Srpske, \$3, 1969 ; Spomenica posvećena stogodišnjici rodjenja Tihomira Djordjevića (Mélanges dédiè à T. Dj., à l'occasion du centenaire de sa naissance), Beograd : SANU, 1971 ; Ijubinkovic (Nenad) “ Život i delo Tihomira R. Djordjevića " (Vie et œuvre de T. R. Dj.), in Djordjevic (T. R.), op.cit., seconde édition, t. 4. Pp. 275-29o (où l'on trouvera quelques autres références).

${ }^{11}$ Djordjević (T. R.), " Amajlije ", in Djordjević (T. R.), op.cit., t. V, 1932 ; puis Djordjević (T. R.), " Hamajlije », in Djordjević (T. R.), op.cit., seconde edition, t. 2. Sur ce terme, cf. Carra de Vaux (B.), "Hamâ'il ", Encyclopédie de l'Jslam, première édition, t. II, 1927. 
Mauvais œil dans la croyance des musulmans d'Ohrid, parue en $1934^{12}$ et, enfin, son célèbre ouvrage intitulé Le mauvais ceil dans la croyance des Slaves du Sud, paru en 1938 et réédité en $1985^{13}$, dans lequel il a repris, complété et systématisé de nombreux passages tirés de ses publications antérieures ${ }^{14}$.

Pour comprendre sa méthode de travail, son bref article sur les talismans est un exemple typique qui mérite d'être analysé en détail. Il y présente, de façon claire et précise, les résultats de ses enquêtes sur le sujet, menés auprès d'un (ou de plusieurs ?) hodja de la ville de Nišn'. L'objet de l'article est brièvement introduit, en deux petits paragraphes qui contiennent cependant beaucoup de données précises :

Chez les Turcs [lire les “ musulmans "], il est une coutume très enracinée, en cas de malheur et en particulier en cas de maladie, d'aller chez les hodja afin qu'ils regardent dans les livres et qu'ils viennent en aide. Les hodja apportent de l'aide de différentes façons ; entre autres, très souvent, au moyen des talismans [amajlije/hamajlije]. Les talismans sont très divers et demandent un examen particulier. Je présente ici, ce que j'ai appris [à ce sujet] pour inciter quelqu'un à examiner ces phénomènes, non seulement chez les Turcs, mais aussi chez nous [lire les " non musulmans "], car ils y sont également très fréquents et très divers.

Chez les hodja viennent chercher de l'aide non seulement les Turcs, mais aussi les chrétiens. À Niš, dans le quartier de Belgrade [u Beograd mahali], il y avait un hodja auprès duquel venaient chercher remède des gens dans le malheur, depuis Aleksinac et même de contrées encore plus éloignées.

Suit une minutieuse description de la préparation des hamajlija "turques" (c'est-à-dire musulmanes), sur laquelle nous devons nous arrêter pour deux raisons. D'une part, du fait qu'elle montre bien la méthode de travail de Djordjević ; d'autre part, du fait que très curieusement, dans ses deux autres

12 Djordjevié (T. R.), "Zle oči u verovanju muslimana u Ohridu ", Glasnik Etnografskog Muzeja u Beogradu, 9, 1934. (En réalité, le volume en question a dủ paraitre très probablement en 1935, si ce n'est encore plus tard.) Cette étude se compose de quatre parties : (a) " Le mauvais œil en général et chez les musulmans d'Ohrid en particulier " (pp. 1-12 et 27-28); (b) "Les hamajlije en général et chez les musulmans d'Ohrid " (pp. 12-28, avec deux planches hors-texte, comportant 28 illustrations) ; (c) La légende sur les "Sept dormants d'Ephèse " (p. 29) ; et (d) un résumé en allemand "Böser Blick in Aberglauben der Muselmanen in Ochrid" (pp. 29-30). Cette étude a évidemment été incorporée ensuite dans le volume paru en 1938 .

${ }^{13}$ Djordjević (T. R.), Zle oči u verovanju Jużnih Slovena, Belgrade : Srpska Kraljevska Akademija, 1938 (seconde édition , Beograd : Prosveta, 1985, avec une importante postface de Ljubinko Radenkovic, pp. 367$380)$.

14 Voir, à titre d'exemple : pour le "mauvais œil", t. 1, 1984, pp. 276-312; pour le "couteau ayant un manche en corne noire" (qui éloigne les mauvais esprits), et les gens appelés "šerbetli" (c'est-à-dire les personnages munis de pouvoirs surnaturels), t. II. pp. 221-222 ; pour les "démons qui s'attaquent aux femmes en couches", t. 4, pp. 106-123, etc.

15 Il s'agissait visiblement d'un hodja albanais, du fait que la lettre arabe lam (donc le -l-y est règulièrement prononcèe et transcrite "à l'albanaise", c'est-à-dire par un -lj- (l "mouillè"). 
études sur la magie (parues à peine quelques années plus tard, en 1934/1935 et en $1938^{17}$ ) qui traitent du mauvais œil et des hamajlija, l'auteur n'a plus du tout abordé le sujet de la confection des talismans de façon aussi détaillée, alors qu'il a repris, notamment dans son célébre volume de 1938, tant d'autres de ses publications antérieures. Dans ce texte, Djordjević relate la séance de fabrication d'un talisman depuis l'arrivée du patient chez le hodja. On comprend que Djordjević a demandé au hodja de lui fabriquer pour lui-même un talisman, tout en lui posant un certain nombre de questions, ce qui lui permet de décrire le processus, à la fois de façon particulière et de façon générale.

Le patient explique d'abord la raison de sa venue. L'ayant écouté, le hodja prend alors le tableau de conversion ${ }^{18}$ des caractères de l'alphabet arabe en chiffres correspondants (tableau qu'il peut d'ailleurs connaître par cœur) et demande le prénom du patient (ou de la personne absente, c'est-à-dire du malade que le visiteur représente) ainsi que celui de sa mère ${ }^{19}$. Au cas où l'on ne connaît pas le prénom de la mère, on utilise celui d'Ève (c'est-à-dire Hawwâ') pour un musulman, et celui de Marie (c'est-à-dire Meryem) pour un chrétien. T. Djordjević ayant pris comme exemple son propre cas, le hodja fait d'abord additionner les chiffres correspondants aux prénoms de Tihomir et de sa mère Jelisaveta, ce qui aboutit dans ce cas précis au chiffre $1778^{20}$. Ensuite, de ce chiffre global, le hodja enlève autant de douzaines qu'il peut, car il y a douze mois dans l'année. Après cette opération, il ne reste forcément qu'un chiffre se situant entre un et douze (ici $1778=12 \times 148+2$ ). Le hodja prend alors un ouvrage dont le titre est " Le livre des étoiles " (Yldız nâme) ${ }^{21}$ qui contient, au début, une liste des douze têtes de chapitres, dont chacune est suivie du nom d'une étoile sous laquelle le patient est né. Le hodja cherche donc ici l'étoile figurant sous

${ }^{16}$ Voir Djordjević (T. R.), " Zle oči u verovanju muslimana u Ohridu " (art.cit.). Cet article sur le mauvais œil dans la croyance des musulmans d'Ohrid était d'ailleurs le fruit d'une enquête effectuée par Djordjević en juillet 1934 à Ohrid, région où l'auteur avait l'habitude de passer une partie de l'été.

17 Voir Djordjević (T. R.), Zle oči u verovanju Južnih Slovena (op.cit.).

${ }^{18}$ Djordjević reproduit ce tableau, mais en caractères cyrilliques, car l'imprimerie ne possédait pas les caractères de l'alphabet arabe. On doit signaler cependant que l'auteur ne mentionne pas à cet endroit l'existence du " grand et du petit abdjad " (sur ce terme, cf. Colin (G. S.), " Abdjad ", EI, nouvelle éd., t. I, 1954).

19 Djordjević rappelle dans une note (dans laquelle il renvoie à un autre endroit de son ouvrage) que, selon les explications de quelques hodja locaux, la demande du prénom de la mère vient du fait que la filiation ne peut être certaine que du côté de la mère, et pas du côté du père. Cela dit, Djordjević rejette cette explication et y voit plutôt (d'après Robertson-Smith (W.), Kinship and Marriage in Early Arabia, London : Cambridge University Press, 1885) une survivance du matriarcat chez les Arabes.

20 L'auteur explique scrupuleusement toute cette opération, notant bien le cas des lettres -e- et -i- de l'alphabet cyrillique que le hodja ne prend pas en compte, alors que pour la lettre -a-finale, il prend la vingt-sixième lettre de l'alphabet arabe, c'est-à-dire la trentième lettre de l'alphabet turc ottoman (donc : le " hâ' " en arabe, le " hé " en turc ottoman, dont la valeur numérique est 5).

${ }^{21}$ Que Djordjević transcrit ici, en caractères cyrilliques, par : "Y'ld'z nâme ». Au sujet de ce type d'ouvrages, voir Massé (Henri), " Fâl-nâma n, EI, nouv. éd., t. II, 1963. 
le titre du chapitre 2, et lit ce qui y est écrit au sujet de la personne née sous ce signe (donc en l'occurrence de notre auteur). Djordjević présente in extenso le texte en traduction serbocroate :

L'homme qui se trouve [donc qui est né] sous cette étoile apprécie les gens cultivés, il est savant et réalise ce qu'il entreprend. Son foyer est prospère, il fréquente des gens [qui sont] bons. Sur le plan matériel il a une bonne situation. Il a des frères dont il a beaucoup d'avantages. Il a père et mère, et d'eux également il tire beaucoup de profit. Il aura plusieurs fils et filles. Il a des maladies : il souffre des jambes et des genoux, mais cela ne dure pas longtemps. Il a une femme et sa femme a bon cœur. Il mourra d'une maladie du cœur. Sur le côté droit il a le signe de la magie (sehir) ${ }^{22}$, et c'est à cet endroit qu'il a des douleurs. Sur le plan matériel tout va bien. Il gagne bien sa vie, mais il dépense très vite. Il se mariera deux fois. Il a une marque à un œil, et aussi sur la jambe [provenant] soit d'une morsure de chien, soit d'une brûlure. Il a beaucoup d'ennemis mais ils sont plus faibles que lui. Le mercredi et le mois de zi l-hidjdje lui conviennent. S'il aperçoit la nouvelle lune, il doit regarder [dans] l'argent et prier Dieu. Il doit porter des vêtements verts. Lorsqu'il se rend auprès de personnes importantes, il doit se placer à leur droite, ainsi sa demande sera exaucée. Il aura peur de la mort à trois moments : à l'âge de sept ans, à l'âge de quatorze ans et à l'âge de quarante-trois ans. S'il surmonte cela, il vivra quatre-vingts ans, sept mois et sept jours. Pour le reste Dieux sait [mieux]. Après cette première opération, le hodja demande au patient s'il veut une hamajlija. Le hodja sait, d'après l'étoile sous laquelle la personne en question est née, quelle hamajlija lui convient. Dans ce cas-là, le patient reçoit une hamajlija et quatre nuska ${ }^{23}$. Si le patient déclare vouloir une hamajlija, alors le hodja la lui confectionne sur une étroite bande de papier en commençant par écrire en arabe, le texte suivant : "Au nom de Suleyman (Salomon) et au nom d'Allah, le Clément, le Miséricordieux ", suivi, toujours en arabe, par un autre texte bref de cinq lignes ${ }^{24}$. Il ajoute ensuite le tableau suivant (qui est évidemment, dans l'édition de Djordjević, toujours en caractères cyrilliques) ${ }^{25}$ :

\footnotetext{
22 Voir Macdonald (D. B.), "Sihr ", El, (première édition) t. IV, 1927 ; et Fahd (T.), "Sihr ", EI, (nouv. éd.), t. IX, 1997.

23 En arabe nuskha (en turc nüsha) veut dire " copie, duplicata, exemplaire, transcription, manuscrit ", mais aussi " amulette, talisman ". Dans les Balkans on dit généralement nuska. Cf. Skaljić (Abdulah), Turcizmi u srpskohrvatskom jeziku (Les turcismes dans la langue serbocroate), Sarajevo : Svjetlost, 1965, p. 496, s.v. " nuskadzija ".
}

24 De ce que j'ai pu déchiffrer, ces quelques lignes écrites en un arabe assez approximatif, et en caractères cyrilliques, contiennent une partie du verset 28 , de la sourate 46 ( al-Ahqqäf $"$ ).

25 Tableau aù l'on lit horizontalement (première ligne) : harplerte [sic ! au lieu de "harflerte" ; littéralement " en lettres arabes "], tâ', sîn ; kâf, hâ, yâa, 'ayn, sâd ; (seconde ligne) : 3 [fois] hâa, 9 [fois] wâw [et en. suite les chiffres]. 


\begin{tabular}{|l|l|l|}
\hline harplerte & Tasin & cef hejeain- sat \\
\hline 3 he waw 9 & 621 & 2 \\
\hline 261 & 293 & 262 \\
\hline
\end{tabular}

Ce ruban de papier est alors plié en triangle, et entouré d'un tissu ciré en trois épaisseurs ; puis le tout est enveloppé dans un tissu sec et cousu à un cordon. L'ensemble, confectionné de cette façon, s'appelle hamajlija et se porte en pendentif autour du $\mathrm{cou}^{26}$.

Les nuska sont également des morceaux de papier comportant un texte écrit en arabe. Dans le cas de la visite de Djordjević chez le hodja, la première nuska comporte un très bref texte commençant par "Bismillahi... "27. Cette première nuska se met dans l'eau et, pendant trois jours, le malade doit boire seulement de cette eau-là. Si le malade est chrétien, pendant ces trois jours il ne peut boire de vin ou de raki (alcool, eau de vie), ni manger de porc, ni d'ail. Le restant de cette eau doit être versé le quatrième jour dans une rivière. Le hodja confectionne les trois autres nuska sur un même morceau de papier qui comporte, en arabe, la triple inscription suivante ${ }^{28}$ :

$$
\frac{492}{919} \text { ha } \frac{1921}{,} \frac{492}{919} \text { ha } \frac{1921}{49} \frac{492}{919} \text { ha } \frac{1911}{} \text {. }
$$

Pendant trois jours, au coucher du soleil, on découpe de ce morceau de papier l'une des nuska, on la jette sur les braises et le malade en inhale la fumée. Après la fumigation, les braises doivent être jetées dans le foyer. Pour chaque personne, la hamajlija et le texte seront différents. De même, les nuska et leur nombre changent. Mais il doit y avoir toujours, cependant, une hamajlija et une nuska à immerger, tandis que le nombre de celles qui servent à la fumigation varie - il peut y en avoir parfois jusqu'à quinze. La fumigation elle-même peut se faire à des moments différents selon les patients. Certains la font le matin, d'autres le soir, et d'autres encore et le matin et le soir. Djordjević précise enfin que les hamajlija se font non seulement en cas de maladie mais aussi contre les sorts (les magies), contre le mauvais œil, contre les armes ${ }^{29}$, etc.

Si le patient est à tel point malade qu'il ne peut venir personnellement chez le hodja, alors quelqu'un d'autre peut venir à sa place chercher la hamajlija. Mais celui-ci doit veiller à ne pas aller avec le talisman aux toilettes, et si il

\footnotetext{
${ }^{26}$ Mais aussi, évidemment, autour du bras ou autrement.

27 Suivent trois lignes de texte en arabe, en transcription en caractères cyrilliques, qui se terminent par une "formule magique" que l'on rencontre souvent, à savoir : "yašafi, yaćafi ("yakâfi"), yameafi ".

${ }^{28}$ Comme plus haut (dans la note 20 ), ici aussi il s'agit de la vingt-sixième lettre de l'alphabet arabe, c'està-dire la trentième lettre de l'alphabet turc ottoman, à savoir le " hè ", dont la valeur numerique est 5 .

29 Dans le texte « contre le fusil ".
} 
est obligé d'y aller avant de l'avoir remis au malade, alors il doit le laisser à l'extérieur afin qu'il ne soit pas désacralisé ou souillé dans les toilettes, auquel cas il perdrait son pouvoir. Pour ses services le hodja ne doit pas demander de rétribution, il doit se satisfaire de ce qu'on lui donne, que ce soit en espèces ou en nature, et seulement de cette façon son travail garde un caractère sacré. Cependant les hodja suivent peu cette règle et, pour la plupart, ils demandent à ètre rétribués.

\section{LE MAUVAIS EIL}

Comme on l'a déjà dit plus haut, l'ouvrage de Djordjević sur la magie chez les Slaves du Sud, paru peu de temps avant la Seconde Guerre mondiale, est encore de nos jours l'unique étude globale sur ce sujet. L'idée de le mettre en chantier lui est probablement venue à l'esprit au cours des années passées en Europe occidentale (1916-1921) où, entre 189o et 1930, c'est-à-dire depuis la parution des travaux de Frazer à ceux de Budge, ont été publiées les toutes premières synthèses sur la magie dans le monde, rédigées par une dizaine de savants ayant des profils et des curiosités scientifiques fort différentes ${ }^{30}$. D'après son titre, sa propre étude devait se limiter uniquement aux Slaves de la péninsule balkanique, mais en réalité elle englobe aussi des renseignements sur la magie chez les Albanais et les Turcs de la région. Par ailleurs, comme le remarque très justement Ljubinko Radenković dans sa postface de la seconde édition de ce livre ${ }^{31}$, les matériaux concernant la magie en Slovénie, par exemple, sont plutōt rares,

$3^{\circ}$ Voici une liste (qui est loin d'être exhaustive) des principaux travaux de ce genre, auxquels Djordjević renvoie régulièrement le lecteur dans ses très nombreuses notes de bas de page : Frazer (James George), The Golden Bough. A Study in Magic and Religion, London : Mac Millan, 189o, 2 vols. ; 1900, 3 vols. ; 1911 1915, 12 vols. ; (trad. fr., Le cycle du rameau d'or, Paris : Geuthner, 12 vols., 1925-1935; réed., Le Rameau d'or, Paris : Robert Laffont, 1981-1984 [et réed. de 1998], 4 vols.) ; Elworthy (Frederic Thomas), The Evil Eye, London, 1895 ; Douttè (Edmond), Magie et religion dans l'Afrique du Nord, Alger : A. Jourdan, 1908 ; réed., Paris : J. Maisonneuve et P. Geuthner, 1984 ; Seligmann (Siegfried), Der böse Blick und Verwandtes. Ein Beitrag zur Geschichte des Aberglaubens aller Zeiten und Völker, Berlin, 1910, 2 vols.; réed. en 1 vol. : Hildesheim / Zürich / New York : Olms, 1985 ; Seligmann (Siegfried), Zauberkraft des Auges und das Berufen (Ein Kapitel aus der Geschichte der Aberglaubens), Hamburg : L. Friedrichsen, 1922 ; Seligmann (Siegfried), Die magischen Heil- und Schutzmittel aus der unbelebten Natur mit besonderer Berücksichtigung der Mittel gegen den bösen Blick. Eine Geschichte des Amulettwesens, Stuttgart : Strecker und Schröder, 1927; Crooke (William), Islam in India or the Qänün-i-isläm. The Customs and Manners of the Musalmans of India... by Ja'far Sharif..., new edition, revised and rearranged, with additions, London : Oxford Univ. Press, 1921 ; Crooke (William), Religion and Folklore of Northern India, Oxford, 1926 ; Westermarck (Eduard), Ritual and Belief in Morocco, London : Mac Millan, 1926, 2 vols. ; Westermarck (Eduard). Pagan Survivals in Mohammedan Civilisation, London, 1933; (trad. fr., Survivances paiennes dans la civilisation mahométane, Paris : Payot, 1935) ; Budge (E. A. Wallis), Amulets and Superstitions, Oxford : Oxford Univ. Press, 1930 ; réed., sous le titre Amulets and Talismans, New York : University Books, 1961, 1970, 1975 ; etc.

31 Voir, Djordjević (T. R.), Zle oči u verovanju Jużnih Slovena (op.cit.), 1985, p. 367. 
et sont encore plus rares ceux concernant les diverses régions de la Bulgarie ce qui est, bien entendu, tout à fait compréhensible. Djordjević en était parfaitement conscient d'ailleurs, puisque dans son introduction il écrivait :

Les données consignées [dans cet ouvrage] sur le mauvais œil chez nous, ne sont pas suffisantes pour pouvoir se faire une idée complète et exacte sur cette question, pas même pour nos régions où elles ont été un peu plus abondamment notées, du fait que le plus souvent elles ont été collectées par des amateurs bien intentionnés, de sorte que leur travail est non seulement insuffisamment détaillé, mais qu'il est même, parfois, également erroné. Enfin, il y a chez nous des régions [entières] où les matériaux concernant le mauvais œil n'ont pas du tout été relevés.

La plus grande partie de ce livre (à savoir trois des quatre chapitres : le premier, le second et le quatrième ${ }^{32}$ ) est consacrée au mauvais œil. Dans le premier chapitre, qui se compose de six sous-chapitres, l'auteur aborde le phénomène du mauvais aeil en général, tout en présentant une grande quantité de détails provenant soit de ses propres enquêtes de terrain, soit des publications d'auteurs locaux parues depuis les dernières décennies du XIX ${ }^{\text {ème }}$ siècle. Tout ce matériau est rangé par thèmes, systématisé et complété par un impressionnant nombre de comparaisons avec des cas similaires observés dans d'autres régions du monde. Les exemples comparatifs sont puisés dans les travaux des principaux spécialistes étrangers, dont Djordjević cite constamment les références, ce qui témoigne de l'éventail de ses lectures. Il présente ensuite (1) un bref historique des études sur le mauvais œil dans le monde en général, le rattachant aux travaux sur la jettatura publiés en Italie. Puis, revenant sur la situation à ce sujet dans les Balkans, il rappelle qu'il existe, dans le cas de l'Église orthodoxe grecque par exemple, des prières spécifiques contre le mauvais œili33. Il examine ensuite (2) la terminologie du mot " urok " (le mauvais cill) et d'autres termes ayant le même sens, utilisés dans les territoires yougoslaves. Les autres parties de ce chapitre traitent très en détail de (3) la façon dont le mauvais œil peut ètre jeté et des jeteurs de mauvais œil (différentes catégories d'humains mais également certains animaux), de (4) qui et quoi est susceptible (et quand et comment) de subir le mauvais œil (hommes, femmes, enfants, animaux, objets inanimés, plantes, travail accompli par quelqu'un), de (5) qui ou quoi est immunisé contre le mauvais œil et, enfin, de (6) quels peuvent être les dommages provoqués par celui-ci. Dans cette volumineuse partie, Djordjević aborde également une quantité d'autres sujets, tels que le phénomène de "magie contagieuse" de Frazer ou le port des amulettes et des talismans, thème sur lequeel il reviendra d'ailleurs de façon détaillée dans le troisième chapitre, comme on va le voir ci-dessous. Il présente également quelques photographies pour mieux illustrer son propos.

${ }^{32}$ Chap. I, « Le mauvais œil " (pp. 1-91 de l'éd. de 1938, pp. 11-107 de l'éd. de 1985) ; Chap. II, « La protection contre le mauvais œil " (pp. 92-116 de l'éd. de 1938, pp. 108-186 de l'éd. de 1985); et Chap. IV, "La guérison du mauvais œil " (pp. 296-324 de l'éd. de 1938 : pp. 314-343 de l'éd. de 1985).

$33 \mathrm{ll}$ cite notamment l'ouvrage de Rodd (Rennel). The Customs and Lore of Modem Greece, London, 1892, p. 160. 
Le chapitre suivant, consacré à la protection contre le mauvais œil est divisé en cinq parties. La première concerne (1) la mise à l'abri du regard d'autrui. Djordjević y étudie les cas des femmes en couches, des enfants en bas àge ${ }^{34}$ et des nouveaux mariés. Il évoque les stratagèmes consistant à donner des réponses inexactes à des questions posées, à cacher le sexe véritable des petits garçons et des petites filles, à changer le parrain des enfants, à couvrir la mariée en la mettant ainsi à l'abri des regards et, par conséquent, aussi du mauvais œil, à mettre des vêtements bizarres et inadéquats, à placer les blessés derrière des rideaux (donc hors du regard d'autrui), à cacher du regard également les ruches et les abeilles. Il mentionne en outre diverses actions à éviter ou, au contraire, à faire : ne pas compter les étoiles, se servir abondamment de l'ail qui est un peu partout considéré comme un excellent moyen de protection, etc. La seconde partie traite du (2) détournement du premier regard d'autrui. Il s'agit d'attirer ce regard par des objets curieux ou totalement inattendus. Ainsi, on placera un tissu de couleur rouge au-dessus du lit d'une femme qui vient d'accoucher ; on portera ostensiblement une cuillère à la ceinture ; ou l'on portera des chaussettes de couleurs différentes. Des stratagèmes similaires, ne faisant pas intervenir un objet, sont aussi possibles. Ainsi, on prévoiera au cours d'un mariage la présence d'une personne qui, par ses pitreries, détournera le regard des invités de la jeune mariée. Le père se couchera dans le lit du nouveau-né, à la place de celui-ci. De façon analogue, il existe des systèmes par lesquels on agit pour protèger les champs, les ruches, les vignobles, les vergers, les maisons d'habitation, etc. La troisième partie concerne (3) l'utilisation des signes et des marques sur le corps afin de détourner le regard d'autrui. Il s'agit de se déguiser de façon frappante au moyen de peintures, de vètements ou de bijoux. La quatrième partie traite de (4) la protection par la malpropreté, par la saleté en général, et plus particulièrement par la salive, les crachats et l'urine. Enfin, la cinquième partie recense (5) les formules de protection, telles : ne budi uroka, " pas de mauvais œil ! ", mašallah, " ce qu'Allah veut ", nazar değmesin, " pas de mauvais œil ! "), utilisées concurrement avec certains termes obscènes ou certaines prières.

Dans le quatrième (et dernier) chapitre de l'ouvrage, Djordjević traite cette fois de la guérison du mauvais œil. Il commence par faire deux remarques d'ordre général. Il cite d'abord un dicton populaire serbe selon lequel " pour chaque maladie existe un remède ". On en conclut que le mauvais œil peut être soigné. Cependant, dans la croyance populaire, on croit aussi fermement que, contre le mauvais œil, les médecins ne disposent pas de remède et que celui-ci ne peut être guéri que par des magiciennes (vračare), ou en tout cas par des

34 Il y a lieu de noter ici que (p. 97. de l'éd. de $1938 ;$ p. 113, de l'éd. de 1985), Djordjevic croyait naïvement que la coutume, ayant eu cours à une certaine époque chez les sultans ottomans, de placer leurs enfants mäles dans des "cages", avait été instauree afin de " les prèmunir contre le mauvais ceil " ! 
femmes âgées. Il en ressortirait donc, selon notre auteur, que, d'une part, presque toutes les actions que l'on entreprend dans le but de la guérison du mauvais œil appartiennent au domaine de la magie et que, d'autre part, la croyance à la nuisance par le mauvais œil est, partout dans le monde, extrêmement ancienne. Ce chapitre contient quatre parties, dont la dernière est subdivisée à son tour en huit paragraphes. La première partie a pour objet (1) la constatation de la maladie. Celle-ci doit être faite pendant qu'il en est encore temps, sinon elle devient incurable. Pour être sûr qu'il s'agit réellement du mauvais œil et non pas d'une autre maladie, les procédés peuvent varier. Chez un enfant, on lèche le front, voire les tempes ou la peau du front entre les sourcils, mais on peut aussi toucher ces mèmes endroits avec un doigt que l'on lèche ensuite. Si le goût est salé, acide ou amer, il est sûr qu'il s'agit du mauvais œil. Chez les adultes, les signes sont les suivants : mal de tête, malaise, sensation de chaud et de froid, baillements incessants, etc., et tout cela sans raison apparente. Il existe aussi des procédés magiques permettant de diagnostiquer la maladie en question. Par exemple, on jette des braises incandescentes dans un récipient contenant de l'eau, soit froide, soit chaude. Si les braises surnagent, il ne s'agit pas du mauvais œil ; mais si elles tombent au fond, celui-ci est diagnostiqué. Il existe de nombreuses variantes à ce procédé. On peut faire la même chose avec du plomb ou de l'ètain fondu, puis interprêter les diverses figures qui viennent de se former au contact du liquide. On peut aussi jeter dans le feu un morceau de sel, que l'on avait auparavant mouillé dans sa bouche, et observer s'il fait du bruit en éclatant dans le feu, ou pas. Il existe aussi d'autres pratiques du mème type, ou plus complexes que les précédentes, et notamment celles où le hodja intervient pour confectionner des écrits et faire des calculs "savants" dans lesquels entrent en jeu les lettres de l'alphabet arabe, avec leur valeur numérique, ce qui permet de multiplier les systèmes et les combinaisons. Des procédés analogues (quand ce n'est pas pratiquement les mêmes, ou avec des variantes) sont décrits dans les deux parties suivantes portants sur (2) la recherche de la personne ayant jeté le mauvais oeil et sur (3) les différentes façons de deviner l'issue de la maladie.

Mais la partie la plus longue de ce chapitre est évidemment la quatrième, c'est-à-dire celle qui est consacrée à la guérison. Intitulée (4) traitement contre la maladie, l'auteur y présente, à l'aide d'une grande quantité de détails, les divers procédés magiques servant à la guérison, tels : l'extinction des braises, le bajanje (pour les humains et pour les animaux), la lecture ou la récitation des prières spéciales (différentes chez les orthodoxes, les catholiques et les musulmans), diverses pratiques magiques (combinant les différents procédés cités), des remèdes et médicaments, ainsi que des méthodes de purification du malade (par l'eau et par les fumigations). Parmi les pages les plus intéressantes, il faut insister surtout sur celles concernant le bajanje, qui est une forme très particulière de magie. Opérée principalement (mais pas exclusivement) par des femmes âgées, elle consiste à 
effectuer des actions magiques les plus variées, en chuchotant à voix mystérieuse et peu audible de brèves incantations appelées basma ou basmica35, dont le sens n'est pas évident à première vue mais qui relève d'un certain nombre de systèmes qui ont une, voire plusieurs, logiques internes ${ }^{36}$. L'ensemble de ses actions sert à éloigner la gêne, le mal, le malheur ou la maladie. La plupart du temps, pour obtenir la guérison, on combine divers procédés magiques, comme cela a été souligné plus haut. Ainsi, par exemple, on combine l'extinction des braises (du plomb ou de l'étain) avec le bajanje, ou bien avec la récitation de telle ou telle prière, ou encore on combine ces trois actions, et d'autres encore, tout en effectuant, simultanément ou successivement (mais au cours de la même séance), des pratiques magiques les plus variées, le tout pouvant être répété un certain nombre de fois (à tel ou tel moment de la journée ou tel ou tel jour de la semaine, à tel ou tel endroit, etc.), selon la prescription du guérisseur.

\section{LES HAMAJLIJE}

Le chapitre le plus volumineux de l'ouvrage est le troisième ${ }^{37}$, dans lequel Djordjević traite des hamajlija (pl. hamajlije), c'est-à-dire des talismans ${ }^{38}$ et des amulettes, dont le rôle est de protéger du mauvais œil, d'annihiler les maléfices causés par celui-ci, par les mauvais esprits ou par des actes magiques divers, mais également de guérir ou de prévenir d'autres maux (maladies, accidents, foudre, etc.).

Ce chapitre se compose de deux parties de longueur très inégale. Tout d'abord, dans une brève introduction, l'auteur analyse savamment l'historique des divers termes servant à décrire ce type d'objet universel, et présente la bibliographie essentielle (étrangère et locale) sur le sujet. Après ces préliminaires, suit une minutieuse description (accompagnée de 72 photos) d'environ cent cinquante hamajlija les plus diverses utilisées dans les territoires yougoslaves. Celles-ci sont classées en quatre catégories, deux d'entre elles étant divisées à leur tour en plusieurs sous groupes. Cette description est précédée d'une introduction qui concerne plus spécifiquement les hamajlija chez les Slaves du Sud. Djordjević y fait plusieurs remarques globales intéressantes. Il note par exemple que le port des hamajlija est nettement plus fréquent dans les régions

\footnotetext{
35 Basma (pl. basme), ou basmica (pl. basmice) qui veut dire "brève basma", ou "petite basma".

${ }^{36}$ Pour les explications des différents systèmes qui régissent les basma, et le "bajanje" en général, voir les ouvrages de Lj. Radenković : Radenković (Ljubinko), Narodna bajanja kod Južnih Slovena (op.cit.) et Radenković (Ljubinko), Simbolika sveta u narodnoj magiji Južnih Slovena (op.cit.).

37 Voir pp. 167-295 de l'éd. de 1938, ou pp. 187-313 de l'éd. de 1985.

$3^{8}$ Soulignons que Djordjević ne voulait pas utiliser ce terme (cf. p. 169 [éd. de 1938], p. 189 [éd; de 1985]) car celui-ci ètait, selon lui, très rarement employé par la population.
} 
sud-slaves où l'influence musulmane a été la plus forte. Il souligne que ce terme arabe et ottoman est attesté en serbocroate dès le XVI ème siècle, et donne des informations très précises sur un certain nombre de coutumes peu connues. Citons, à titre d'exemple, celles concernant la réutilisation des hamajlija après le décès de leur propriétaire, ou la très curieuse façon d'utiliser comme hamajlija un petit bout de placenta d'un nouveau-né (ou d'un tout petit morceau d'un arbre [?] ${ }^{39}$, de la taille d'un grain de blé) que l'on introduit sous la peau du bras droit (ou sous l'aisselle) de l'enfant.

Comme je viens de le signaler, l'auteur classe l'ensemble des hamajlija étudiées en quatre catégories, à savoir : les hamajlija populaires, chrétiennes, musulmanes et mixtes.

Concernant la première catégorie, celle des hamajlija populaires, il note qu'elles existaient déjà à l'époque du paganisme et que l'on peut les diviser à leur tour en trois sous groupes, à savoir : les hamajlija minérales, végétales et animales. Leur force proviendrait de leur caractère propre (la dureté, le tranchant, le piquant, la douceur, l'aigreur, la couleur, etc.). Il existerait également un quatrième sous groupe, celui des hamajlija composites, formées d'un élément minéral et d'un élément végétal par exemple ou d'un élémént végétal et d'un élément animal, ou encore d'un élément minéral et d'un élément animal.

Du fait que les minéraux sont beaucoup moins connus de la population que les plantes ou les animaux, les hamajlija minérales sont nettement moins nombreuses. Djordjević n'en cite d'ailleurs que six (le sel, le fer, l'or, l'argent, le mercure, les "pierres de foudre" [ou "pierres de tonnerre"] et, enfin, différentes sortes de pierres précieuses et semi-précieuses), au sujet desquelles il fournit de nombreux exemples provenant des diverses régions du monde, y compris du Sud-Est européen.

Les hamajlija végétales sont beaucoup plus répandues. Elles sont employées pour des infusions, des fumigations, des bains du corps, ou portées telles quelles, parfois cousues sur les habits. Djordjević en cite une trentaine, parmi lesquelles, l'ail, l'oignon, le bois d'if, la rue, la nigelle, la ronce, l'aubépine, la pomme de pin, la noix de galle, une herbe rougeâtre appellée "cœur de lapin" qui est censée avoir été "vomie par des sorcières", la mauve, le romarin, le poivre, le bois résineux, le cornouiller, une herbe contenant du poison appelée "ludinjak" qui a le pouvoir de rendre fou, le chêne, le gui, la fougère, la bardane, mais aussi les produits faits des végétaux, comme le pain, des fils de coton, ou une chemise qui a été tissée en une seule nuit... Tous ces exemples sont accompagnés de commentaires et de références.

39 L'auteur écrit “ česno drvo ", ce que l'on pourraît traduire, peut-être, par " l'arbre sacré ", (časno drvo), ce qui n'est qu'une supposition. Mais à un autre endroit de son livre (voir p. 249 de l'éd. de 1938, et p. 312 de l'éd. de 1985), il spécifie qu'il s'agirait en fait de " crni glog ", donc de l'aubépine "noire". 
Les hamajlija animales sont encore plus répandues et Djordjević en cite un très grand nombre. Il s'agit, d'une part, d'objets faits à partir de certaines parties du corps humain, le plus souvent un petit morceau du placenta d'un nouveau né ou de son cordon ombilical, mais également d'autres parties de corps, dont le choix et l'éventail sont parfois très surprenants. Il s'agit, d'autre part, de parties de corps d'animaux de toute sorte : os creux de chien, œuf de loutre, croupion de canard, queue de serpent à sonnettes, morceau de la peau de lapin, queue de lapin ou de poisson, dent de loup, de sanglier ou d'ours, morceau de corne de tel ou tel animal, patte de taupe, griffe d'aigle ou d'épervier, parties du corps de la chauve-souris, escargot, coquillages, etc.

À côté de ces hamajlija que Djordjević qualifie de "populaires ", il y a les hamajlija chrétiennes, dont la puissance, contrairement aux précédentes, ne provient pas de leurs qualités propres mais de leur rapport au christianisme. En introduction, l'auteur décrit fort bien, et en peu de mots, la complexité et l'évolution des rapports entre magie et christianisme. On assiste d'abord, au Concile de Laodicée qui eut lieu vers 343 , à la condamnation la plus absolue de l'usage de talismans et de la magie en général. Mais, dès les débuts du Vème siècle, l'Église cède peu à peu le pas, pour aboutir à des "arrangements" tacites, puis à une reconnaissance sous entendue d'un certain nombre d'objets, qui font depuis office de ce que l'on peut appeler les hamajlija chrétiennes. L'auteur analyse ensuite longuement ces différentes sortes d'objets et de reliques, en commençant évidemment par la croix, les images de la Vierge, du Christ, des anges, des grands Saints, des martyrs de l'Église, ainsi que les extraits tirés des Écritures, reproduits sur différents supports, comme le cuir, le papyrus, la pierre et le papier. Il s'attarde sur leur description (avec des illustrations), ainsi que sur différents types de médailles, de médaillons et de petites icônes représentant la Vierge à l'enfant, le baptême du Christ dans le Jourdain, Saint Georges terrassant le dragon, etc. Ces objets sont portés sur soi, tels quels, de façon visible, ou au contraire cousus dans les vêtements, donc de façon invisible, voire dans de petites boîtes en métal que l'on porte, sous forme de pendentifs autour du cou ou du poignet. Il examine également le cas des bannières, des oriflammes et des enseignes d'églises, ainsi que de très nombreux objets relatifs au rituel religieux, et de ce fait considérés comme "saints" (ossements et reliques, mais aussi encens, pain de la communion, certaines plantes comme le basilic, etc.).

Enfin, il place dans une catégorie à part les zapisi (ou "écritures"). Il s'agit de morceaux de papier sur lesquels sont généralement inscrites des prières (souvent apocryphes), des passages tirés des Évangiles et des livres saints, des suppliques ou incantations diverses et, parfois, des menaces. Ces zapisi sont souvent écrits à la main, par des prêtres ou par leurs sacristains, mais il en existe également sous une forme imprimée (et qui sont d'ailleurs beaucoup plus souvent demandées par la population). Djordjević ajoute à ce sujet que les 
zapisi en question ont un caractère plus magique que religieux. Ces zapisi sont confectionnés non seulement pour les humains mais aussi pour les animaux domestiques. En ce qui concerne les hommes, ceux destinés à la population orthodoxe sont écrits en caractères cyrilliques, alors que les zapisi pour les catholiques sont en caractères latins. Dans les toutes dernières pages de cette partie, l'auteur traite de l'utilisation dans un but médico-magique du fameux "sceau de Salomon" et des divers livres saints qui sont portés comme hamajlija, le plus connu étant intitulé "Le rêve de la Sainte mère de Dieu ", (San Svete Bogorodice), dont le texte est tiré d'une hrisovulja, "chrisobulle" $4{ }^{\circ}$ en provenance du Mont Athos.

La troisième catégorie est celle des hamajlija musulmanes, sujet particulièrement complexe auquel Djordjević consacre plus d'une cinquantaine de pages et de nombreuses illustrations. Dans son préambule, il décrit comment elles sont apparues dans les différentes régions étudiées, insistant sur la situation fondamentalement différente de la magie (sihr/sihir) dans l'islam et le christianisme. Il analyse sa pénétration dans les territoires yougoslaves, y compris chez les populations non musulmanes du pays. Procédant ensuite de la même façon que pour la catégorie précédente, il divise les hamajlija musulmanes en deux groupes distincts : les hamajlija populaires et religieuses. Dans son analyse, il fait ressortir, de façon commode, les analogies et les divergences par rapport aux hamajlija chrétiennes.

On remarque ainsi dans le groupe des hamajlija populaires minérales musulmanes, la mention de quelques autres minéraux, comme l'ammoniac (nišador), une sorte d'asphalte ou de goudron mou nommé ljaden, différentes sortes de pierres (turquoise, "pierre bleue" dite gök tașı, perle de verre bleue, appelée mavi boncuk ou nazar boncuğu), des petites épées, massues et fusils en miniature (faites en argent ou en autres métaux) que l'on fixe sur les vêtements ou sur les bonnets des enfants, etc.

En ce qui concerne les hamajlija populaires végétales musulmanes, il mentionne par exemple l'ail, ainsi que les graines noires de la plante appelée çurek otu (nigella damascena), avec lesquelles on saupoudre les simit (petits pains en forme d'anneau) et le pain fait à l'occasion des grandes fêtes religieuses musulmanes comme le bayram (bayram ekmeği), graines que l'on peut aussi envelopper dans un petit morceau de toile qui sera cousu ensuite sur les vêtements. Dans cette mème catégorie, il mentionne aussi une sorte de noix de galle, dite mazi (ou mazija), avec laquelle les femmes musulmanes se colorent les cheveux.

Enfin, pour ce qui est des hamajlija populaires animales musulmanes, Djordjević mentionne, en plus de celles qui ont été citées au sujet des hamajlija chrétiennes : la queue de scorpion, l'œil de loup, un morceau de cœur de loup, voire des hamajlija faites en forme d'œil, et que l'on incruste sur des sab- 
res ou d'autres objets. Un genre particulier est celui des hamajlija représentant la main humaine ou les cinq doigts de la main (faites en or, en argent ou en un autre métal), la patte d'un animal, ainsi que des cloches, des clochettes et des hochets que l'on accroche au cou, sur les harnais et sur les brides des animaux domestiques à quatre pattes. Djordjević ne classe d'ailleurs pas ces objets dans le groupe des hamajlija minérales, considérant que leur force contre les maléfices ne provient pas des matériaux dont ils ont été faits, mais du son que ces objets produisent, censé chasser les mauvais esprits.

L'auteur s'attarde ensuite longuement sur les hamajlija religieuses musulmanes. Il rappelle tout d'abord leurs origines, puis décrit les nuska (nuskha ou nüsha, donc les zapisi ou "écritures") et la manière de les confectionner (ce qui est surtout fait par les derviches et les hodja), ainsi que les pratiques et les croyances diverses les concernant, tout en citant - selon son habitude - un grand nombre d'exemples accompagnés de références. Il mentionne notamment les nombreux cas où les chrétiens viennent chercher des nüsha chez les religieux musulmans et vice versa. Il fait également état de médaillons faisant office de hamajlija, sur lesquelles on voit parfois des symboles religieux chrétiens et musulmans se côtoyer (comme par exemple la croix et l'inscription mașallah). Djordjević mentionne également l'existence des hodžinska hamajlija, petit carnet de notes servant de vade mecum aux hodża pour composer des zapisi, et des ćitap hamajlija, à savoir des Corans en miniature, portés en pendentif dans une petite boîte en métal. Il évoque aussi les divers objets en tissu, en cuir ou en métal servant à contenir les zapisi sur papier, tels que mahfaza (ou hajmali mahfaza), pazvant (ou pazubent, pazbent, du persan bâz u bend), dilbagija (dilbağı), et ename (ou enâm-hamajlija).

Enfin, la quatrième et dernière catégorie de hamajlija définie par Djordjević est celle des hamajlija mixtes, à savoir celles que l'on peut considérer comme étant à la fois des hamajlija populaires et des hamajlija religieuses. L'auteur examine d'abord, très rapidement, le cas du placenta de nouveau-né qui, chez les chrétiens, peut revêtir un pouvoir quasi religieux par le truchement de sa "liturgisation" préalable. En effet le pope (voire son sacristain) place le placenta près de lui (donc quelque part dans l'autel), au cours des offices religieux, durant six semaines, avant de le rendre à la famille. Bien entendu, chez les musulmans des régions étudiées, le procédé est tout à fait différent. Chez eux on fait d'abord sécher le placenta, sur lequel le hodja va écrire ensuite un zapis (talisman). Djordjević donne également quelques autres exemples similaires : le pain de la communion avec un peu de sel et un grain de blé, la patte de taupe, les dents de sanglier, l'ail, le mercure, voire un zapis fait par le hodja ou par le pope ${ }^{41}$.

$4^{1}$ Pour compléter la présentation de cette étude de Djordjević, il y a lieu d'ajouter que le volume se termine par une brève version de la légende des Sept dormants d'Éphèse et par un copieux index qui facilite son utilisation. 


\section{CONCLUSION}

On comprendra aisémént je pense, d'après ce qui précède, que l'apport de Tihomir Djordjević à l'étude de la magie chez les musulmans, et les non musulmans, des Balkans a été, et reste encore aujourd'hui, essentiel et incontournable et qu'il doit servir de base aux travaux à venir dans ce domaine. Cela étant dit, cette constatation appelle aussi deux remarques de simple bon sens. Il faut rappeler que Djordjević n'était évidemment pas un "orientaliste", c'està-dire un islamisant ou islamologue et que cela réduisait donc ses possibilités d'approcher de près certains problèmes ou complexités, concernant la magie dans le monde musulman médiéval et moderne en général, et dans le monde musulman du Sud-Est européen en particulier ${ }^{42}$. En outre, on constate qu'il a, curieusement, très peu utilisé le seul livre existant à son époque sur la magie chez les derviches du Kosovo et de la Macédoine, celui de Gliša Elezović, alors que celui-ci traite de ce sujet sur une bonne soixantaine de pages 43 .

Je terminerai en rappelant que, de son côté, pour des mobiles extra-scientifiques (et avant tout politiques), l'œuvre monumentale de Djordjević a été ignorée dans les publications des deux principaux spécialistes de la magie musulmane (et non musulmane) en Bosnie-Herzégovine, Stanko Sielski et Muhamed Garčević, parues en 1942 à Zagreb, Banja Luka et Sarajevo. Il a cependant été honoré par une seconde édition de son opus magnum sur le mauvais œeil, parue à Belgrade en 1985, dans laquelle on trouvera une Postface de Ljubinko Radenković, qui contient une rapide presentation de l'ouvrage ainsi que des réflexions théoriques sur le problème du mauvais œil et les incantations (bajanje).

42 Il avait utilisè, bien entendu, l'Encyclopédie de l'Islam, et avait consulté à plusieurs reprises son collègue de l'Université de Belgrade, Fehim Bajraktarević, qu'il remercie vivement à ce sujet dans l'introduction de son livre.

43 Voir घlezović (Gliša), Derviški redovi muslimanski. Tekije u Skoplju (Les ordres musulmans des derviches. Les tekke de Skoplje), Skoplje, 1925, Pp. 32-95 (dont le texte avait paru auparavant dans plusieurs numéros des périodiques Stara Srbija et Crkva i život, de Skoplje). Djordjević n'utilise cet ouvrage qu'à dewx reprises (pp. 242 et 275 de l'éd. de 1938, et pp. 266 et 295 de l'éd. de 1985). 